\title{
Age Trends in Visual Exploration of Social and Nonsocial Information in Children with Autism
}

\author{
Jed T. Elison ${ }^{1,2}$, Noah J. Sasson ${ }^{4}$, Lauren M. Turner-Brown ${ }^{2,3}$, Gabriel Dichter ${ }^{2,3}$, and \\ James W. Bodfish ${ }^{2,3}$ \\ ${ }^{1}$ Department of Psychology, University of North Carolina at Chapel Hill (UNC) \\ ${ }^{2}$ Carolina Institute for Developmental Disabilities, UNC \\ ${ }^{3}$ Department of Psychiatry, UNC \\ ${ }^{4}$ The University of Texas at Dallas, School of Behavioral and Brain Sciences
}

\begin{abstract}
Because previous studies of attention in autism spectrum disorders (ASD) have been restricted in age range examined, little is known about how these processes develop over the course of childhood. In this study we examined cross-sectional age effects on patterns of visual attention to social and nonsocial information in 43 typically developing children and 51 children with ASD ranging in age from 2 to 18 . Results indicated a sharp increase in visual exploration with age and a decrease in perseverative and detail-focused attention for both groups of children. However, increased age was associated with greater increases in visual exploration for typically developing children than for those children with ASD. The developmental differences were most pronounced for attention to certain nonsocial stimuli as children with ASD demonstrated a disproportionate attentional bias for these stimuli from very early in life. Disproportionate visual attention to certain nonsocial objects relative to social stimuli in ASD spanned from early to late childhood, and thus may represent both an early and a persistent characteristic of the disorder.
\end{abstract}

\section{Keywords}

autism; attention; visual exploration; development; eye tracking

\section{Introduction 1}

Children with autism spectrum disorders (ASD) preferentially orient to certain nonsocial information (Sasson et al., 2008; Pierce et al., 2011) relative to social information (see also Klin et al., 2009). Extended experience with certain categories of information (i.e., nonsocial information) over time is likely to have effects on brain development and the specialization

\section{(C) 2011 Elsevier Ltd. All rights reserved.}

Correspondence should be addressed to: Jed Elison, Ph.D. Caltech-HSS, 1200 E. California Blvd., MC 228-77, Pasadena, CA 91125 , Phone: 626-395-8982, Fax: 626-793-4681, jelison@caltech.edu.

Conflict of Interest:

The authors declare no conflicts of interest.

Publisher's Disclaimer: This is a PDF file of an unedited manuscript that has been accepted for publication. As a service to our customers we are providing this early version of the manuscript. The manuscript will undergo copyediting, typesetting, and review of the resulting proof before it is published in its final citable form. Please note that during the production process errors may be discovered which could affect the content, and all legal disclaimers that apply to the journal pertain.

${ }^{1} \mathrm{ASD}=$ Autism Spectrum Disorders; TD=Typically Developing; VET=Visual Exploration Task; HAI=High Autism Interest; LAI=Low Autism Interest; PIQ=Performance Intelligence Quotient 
of dedicated neural circuitry (Gauthier et al., 2000; Johnson, 2001). Thus, ASD may involve a difference in the balance of attention between social and nonsocial aspects of everyday experience which may over time manifest behaviorally as excessive nonsocial interests and behaviors coupled with diminished social interests and behaviors.

We recently developed a passive viewing eye-tracking task (the "Visual Exploration Task" VET) that can be used to measure the balance of attention between social and nonsocial information (Sasson et al., 2008). In a follow-up study we demonstrated that the VET could be easily administered to children of varying ages and cognitive abilities (Sasson et al., 2011). In the VET, a series of complex arrays containing images varying in categorical social and nonsocial content is presented and participants are permitted to explore each array in any manner they choose (i.e. there are no instructions). Eye-tracking technology is used to derive three discrete attentional variables that are conceptually linked to potential aspects of the autism phenotype: exploration (number of images fixated), perseveration (average fixation time per image explored), and detail-oriented visual scanning (number of discrete fixations per image explored).

Compared to children who are developing typically, children with ASD tend to explore fewer images overall, perseverate to a greater degree on the images they do explore, and are more detail-oriented in their inspection of individual images. Group differences are most pronounced for high-autism-interest (HAI) nonsocial images, a subcategory of nonsocial images that represent a diverse range of objects related to circumscribed interests as reported in South et al., (2005). Children with ASD disproportionally explored, perseverated and inspected in detail these object types relative to other image categories. This heightened salience of certain non-social aspects of the environment may have developmental repercussions, as the development of neural specialization related to many social information processing abilities are experience-dependant and may be adversely affected when the quantity and quality of visual experience with social stimuli is abnormal (Leppanen \& Nelson, 2009; Sasson, 2006; Schultz, 2005).

In general, findings from the VET are consistent with other studies that have examined visual preferences in autism (Klin et al., 2009; Pierce et al., 2011), collectivity suggesting that either social information is less salient, or that nonsocial information is more salient for young children with ASD. While these studies have established early abnormalities in visual attention as a robust cognitive phenotype in ASD, to date there have been no studies of visual attention in ASD that have examined age trends in attentional performance across a broad range of ages. Thus, it is currently unknown how these visual attention patterns change over time. An early bias toward nonsocial information could potentially canalize across development, thereby increasing the bias over time. Alternatively, the bias toward nonsocial information could attenuate over time due to accumulated exposure to the ubiquitous presence of social information encountered on a daily basis, particularly during the school-age years. Evidence supporting either of the hypotheses has the potential to elucidate optimal timing and the precise target for directed intervention.

The aim of the present study was to determine the effect of age on exploration, perseveration and detailed-oriented scanning during the VET in a cross-sectional sample of 2 to 18 yearold typically developing children and children with autism. We hypothesized that age would account for increases in visual exploration patterns as assessed by our three eye tracking measures in typically developing children, but that children with autism would show less of an age effect. To examine this question we (a) combined our two previous samples of participants from our existing VET studies (school-age from Sasson et al., 2008; preschool age from Sasson et al, 2011), and (b) included new data from 20 additional participants in order to form a more complete age distribution spread proportionately from 2 to 18 years. 
Although this in part includes re-analysis of existing data, in neither of the previous studies was the age range sufficient to explore age trends. Thus the combined + new VET samples permitted a novel analysis aimed at an unexamined area of visual attention in ASD.

\section{Methods}

\subsection{Participants}

A total of 94 children participated in this study. Data from 74 of the 94 participants were drawn from two previous studies (Sasson et al., 2008; Sasson et al., 2011), while the remaining 20 are unique to the present study. The primary purpose of Sasson et al. (2008) and Sasson et al. (2011) was to examine categorical group differences in voluntary visual attention patterns between typically developing (TD) children and children with ASD. In contrast, the current study focuses on continuous age-related changes in attentional patterns, an approach that was only possible after combining across samples from both previous studies and including 20 new participants to complete a continuous distribution from 2 to 18 years of age. To achieve this distribution, a total of 31 additional participants were recruited, of which seven typically developing children (TD: all male; mean age: 175.42 months, SD: 14.73) and four children with ASD (all male; mean age: 143.50 months, SD: 29.01) were excluded because of missing or low quality data resulting from inaccurate eye tracking calibrations, or from non-compliance. Experimental procedures for the new participants were identical in all respects to those tested previously. The final sample of 94 children consisted of 51 with ASD (48 males, 3 females; mean age: 111.51 months (SD: 43.22); range: 32 to 207 months) and 43 who were TD (39 males, 4 females; mean age: 98.47 months (SD: 50.33); range: 25 to 194 months). The ASD and TD groups were not statistically different in sex $\chi^{2}=0.40, p=.53$ or age $(t(92)=-1.35, p=.18)$, but PIQ did differ between groups for the 78 individuals (48 ASD, 30 TD) for which PIQ information was available $(t(76)=4.50, p<.01)$. Age and PIQ were not correlated $(r=.14, p=.22)$. Demographic and clinical features of participants can be found in Table 1.

No participant had a history of seizure disorder, or any acute medical or genetic conditions. All had normal or corrected-to-normal vision. TD children were recruited through an email message sent to UNC faculty and staff, and were excluded if they had a history of developmental or psychiatric disorder, were taking psychotropic medication, or had an immediate family with an ASD diagnosis. ASD children were recruited from the UNC Autism Research Registry in conjunction with regional TEACCH (Treatment and Education of Autistic and related Communication-handicapped CHildren) clinics. To be included in this registry, children are required to have a DSM-IV diagnosis of ASD, as determined by a licensed clinician with expertise in autism diagnosis and assessment. Forty-seven of the 51 ASD children had diagnoses confirmed with the Autism Diagnostic Observation Schedule (Lord, et al, 2000) and/or the Autism Diagnostic Interview-Revised (ADI-R; Lord et al., 1994; Risi, et al., 2006). The four ASD participants who had not received diagnostic confirmation using these instruments were all young children (mean age: 44 months; SD: 10.23) with diagnoses of Autistic Disorder that were not questioned by TEACCH clinicians (see Sasson, et al., 2011). The Biomedical Institutional Review Board at the UNC-Chapel Hill School of Medicine approved the protocol of this study. A legal guardian of each participating child provided informed written consent and children 6 years of age and older provided verbal and written assent.

\subsection{Procedure}

Visual Exploration Task-The VET consists of 12 static arrays, each comprised of 24 individual color images distributed around an empty space in the center of the array where all scanning patterns began. Six of the arrays are "social + object arrays" (i.e., they contain 
images of people and objects) and six are "object only arrays" (i.e., they only contain images of objects). Examples can be seen in Figure 1. All social images depicted males and females of various ages and ethnicities displaying clearly visible happy expressions. Half of the social images presented the face in isolation, and half included the full head and body. Of the object images, half were selected to be of "High Autism Interest" (HAI) and half to be of "Low Autism Interest" (LAI). HAI objects were chosen from nine non-social categories that previous research has indicated are common targets of circumscribed interests in ASD: trains, planes, vehicles (e.g., cars and trucks), computer equipment, home electronics, blocks, road signs and sporting equipment (South, et al., 2005). LAI objects were chosen from nine categories that have not been found to be common targets of circumscribed interests in ASD: clothing, furniture, food, plants, hats, gloves, school supplies, bathroom supplies and bags.

Three of the "social + object arrays" contained social images paired with HAI objects and 3 contained social images paired with LAI objects. Because the VET is designed to measure the relative salience of images related to circumscribed interests as they compete for visual attention with other social or nonsocial information, arrays containing only social images were not included in the procedural design. The combination of social + HAI or social + LAI images was counterbalanced across a set of image-type ratios $(5: 1 ; 3: 3 ; 1: 5)$ (e.g., 20 social + 4 HAI or 12 social + 12 HAI or 4 social + 20 HAI). The object only arrays contained HAI objects paired with LAI objects. Two versions of each image-type ratio (5:1; 3:3; 1:5) (e.g., $20 \mathrm{HAI}+4 \mathrm{LAI}$ or $12 \mathrm{HAI}+12 \mathrm{LAI}$ or $4 \mathrm{HAI}+20 \mathrm{LAI}$ ) were used to produce 6 total "object only arrays" that counterbalance with the 6 "social + object arrays".

Testing Procedure-All participants were tested in a research laboratory on the campus of UNC-Chapel Hill. The VE task was presented on a Tobii 1750 eye-tracker, which integrates eye-tracking equipment within a $17^{\prime \prime}$ diagonal display. Participants sat approximately $60 \mathrm{~cm}$ from the display (visual angle, $\sim 32^{\circ}$ ), and data was recorded at a sampling rate of $50 \mathrm{~Hz}$. After a brief 5-point calibration procedure, participants were instructed to view a series of pictures. The 12 arrays were then presented one at a time for $10 \mathrm{~s}$ each in one of three pseudorandom orders. Before each array was presented, a central crosshair appeared in the middle of the screen to reorient the participant and ensure that each scanning pattern began at the same point for each participant and each array. Images were never displayed in this center location.

\subsection{Statistical Analysis}

Visual attention patterns of participants were examined by analyzing the distribution, location and duration of fixations. Fixations were defined as gaze remaining within a 30 pixel radius $\left(\sim .80^{\circ}\right.$ of visual angle) for a minimum of $100 \mathrm{~ms}$. As detailed in Sasson, et al. (2008) and Sasson, et al. (2011), three dependant variables, each related to attentional and perceptual characteristics of the autism phenotype, were assessed: exploration (the number of discrete images fixated), perseveration (the duration of total fixation time per image fixated), and detail-orientation (the total number of discrete fixations per image fixated). While these variables correspond to previously reported conceptually distinct aspects of the autism phenotype (e.g., exploration, Pierce \& Courchesne, 2001; perseveration, Landry \& Bryson, 2004; detail-orientation, Happe \& Frith, 2006), they are moderately interrelated when assessed within the VET (Sasson, et al., 2008), as greater perseveration and detailorientation on this task can reduce exploratory behavior. In the current study, exploration was negatively associated with perseveration $(r=-.42)$ and detail-orientation $(r=-.58)$, while perseveration and detail-orientation were positively associated with one another $(r=$. $55)$. 
Previous reports of the VET focused on categorical differences between ASD and TD on the three outcome measures (Sasson et al., 2008; 2011). In contrast, the current study examined age-related changes in the allocation of overt visual attention on this task, and assessed whether this pattern differed between children with and without ASD. Characterizing the effect of age, as a continuous variable, on performance level could potentially elucidate the nature of attentional deficits in ASD (i.e., whether they canalize over time or whether some compensatory mechanism(s) normalizes attentional performance over time), and therefore inform directed treatment. To address the hypothesis that age would be related to the three dependant variables (exploration, perseveration, detail-orientation) across the entire task, and to assess whether this pattern would differ between groups, separate hierarchical regressions for each dependant variable were conducted in two steps: first, age was entered as a continuous predictor and group as a categorical predictor; next, the age $\times$ group interaction term was entered to determine if it predicted each dependant measure above and beyond the independent predictors. To determine if age-effects varied as a function of image type, separate hierarchical regressions using the same sequence of predictors were then conducted independently on social images, HAI objects and LAI objects. Additionally, to explore the possibility that PIQ may have affected these visual patterns, we conducted supplemental analyses on the portion of the sample in which PIQ information was available (all children > 62 months; $\mathrm{n}=72 ; 76.6 \%$ of the total sample). Separate hierarchical regressions for exploration, perseveration and detail-orientation were conducted in the same steps as outlined above, but now with PIQ entered into the model first. This enabled us to determine whether PIQ predicts performance on these three dependent measures, and more importantly, whether age and group account for a significant portion of the variance in each above and beyond any effects of PIQ.

While the ASD and TD groups did not differ in the amount missing data (i.e., eye blinks and gaze time off screen) $(\mathrm{t}(92)=.71, \mathrm{p}=.46)$, missing data did significantly decrease with age $(\mathrm{r}=-.48, \mathrm{p}<.01)$. To control for these age effects in missing data, the dependent measures were calculated as a ratio of gaze-time onscreen. We used an alpha level of 0.05 for all statistical tests.

\section{Results}

To examine age effects in our sample, we computed correlations between age and visual attention measures for both TD and ASD children (see Table 2). As predicted, age was significantly correlated with all three measures for both groups; exploration increased with age while perseveration and detail orientation decreased.

Next, regression analyses were used to determine whether age and group uniquely predicted exploration, perseveration and detail-orientation, both overall across the task, and broken down by image type. Results of the regression analyses are detailed below and summarized in Table 3. Finally, supplemental regression analyses on three dependant measures were conducted with the 72 children who had PIQ information to examine whether level of cognitive functioning affected any reported findings.

\subsection{Exploration}

The combination of age and group significantly predicted approximately $46 \%$ of the variance in exploration across the entire task (adjusted $\mathrm{R}^{2}=0.462 ; \mathrm{F}(2,91)=40.91, \mathrm{p}<$. $001)$, with age $(\mathrm{p}<.001)$ and group $(\mathrm{p}<.001)$ each significantly contributing a unique portion of the variance. The addition to the model of the age $\times$ group interaction contributed an additional $3 \%$ to the total variance $\left(\mathrm{R}^{2}\right.$ change $\left.=0.028\right)$, and the overall model remained statistically significant (adjusted $\mathrm{R}^{2}=0.485 ; \mathrm{F}(3,90)=30.14, \mathrm{p}<.001$ ). More importantly, the incremental gain in variance was also significant $(F(1,90)=5.00, p=.028)$, indicating 
that the age $\times$ group interaction variable independently accounted for an additional $3 \%$ of the variance in exploration above and beyond that of age and group alone (see Figure 1).

For social images (extracted from social arrays), age and group together significantly accounted for approximately $40 \%$ of the variance in exploration (adjusted $\mathrm{R}^{2}=0.401 ; \mathrm{F}(2$, $91)=32.16 . \mathrm{p}<.001)$, with age $(\mathrm{p}<.001)$ and group $(\mathrm{p}<.001)$ both contributing a unique portion of the variance. The incremental gain in variance after adding the age $\times$ group interaction to the model $\left(\mathrm{R}^{2}\right.$ change $\left.=0.023\right)$ approached significance $(\mathrm{p}=.058)$. On the object only arrays, age and group together significantly predicted approximately $33 \%$ of the variance in the exploration of HAI objects $(\mathrm{F}(2,91)=23.43, \mathrm{p}<.001)$, with age $(\mathrm{p}<.001)$ but not group $(\mathrm{p}=.14)$ significantly contributing a unique portion of the variance. Adding the age $\times$ group interaction contributed an additional $5 \%$ to the total variance $\left(\mathrm{R}^{2}\right.$ change $=$. 049), and the overall model remained statistically significant $(\mathrm{F}(3,90)=19.13, \mathrm{p}<.001)$. The incremental gain in variance was significant $(\mathrm{F}(1,90)=7.29, \mathrm{p}=.008)$, indicating that the age $\times$ group interaction variable accounted for a significant additional portion of the variance in the exploration of HAI objects above and beyond age and group alone. Age and group together significantly accounted for $30 \%$ of the variance in the exploration of LAI objects (adjusted $\left.\mathrm{R}^{2}=0.300 ; \mathrm{F}(2,91)=20.93, \mathrm{p}<.001\right)$, with age $(\mathrm{p}<.05)$ and group $(\mathrm{p}<$. $001)$ both uniquely contributing. Adding the age $\times$ group interaction predicted an addition . $1 \%$ of variance to the model, and was not significant.

\subsection{Perseveration}

Together, age and group significantly accounted for approximately $40 \%$ of the variance in perseveration across the entire task (adjusted $\mathrm{R}^{2}=0.404 ; \mathrm{F}(2,91)=32.56, \mathrm{p}<.001$ ), with age $(\mathrm{p}<.001)$ but not group $(\mathrm{p}=.10)$ contributing a statistically significant unique portion of the variance. The incremental gain in variance after adding the age $\times$ group interaction to the model was minimal and not significant $\left(\mathrm{R}^{2}\right.$ change $\left.=.001\right)$.

For social images (extracted from social arrays), age and group combined to significantly predict approximately $19 \%$ of variance in the perseveration (adjusted $\mathrm{R}^{2}=0.186 ; \mathrm{F}(2,91)=$ $11.63, \mathrm{p}<.001)$, with age $(\mathrm{p}<.01)$ but not group $(\mathrm{p}=.77)$ significantly contributing. The addition $1.4 \%$ of variance predicted by adding the age $\times$ group interaction to the model was not significant. On the object only arrays, age and group together significantly predicted approximately $39 \%$ of the variance in the perseveration on HAI objects (Adjusted $\mathrm{R}^{2}=$ $0.391 ; \mathrm{F}(2,91)=30.89, \mathrm{p}<.001)$, with age $(\mathrm{p}<.001)$ and group $(\mathrm{p}=.05)$ each uniquely contributing to a portion of the variance. The addition of the age $\times$ group interaction only explained an additional $.3 \%$ of the variance, and was not significant. Age and group combined to significantly predict $19 \%$ of the variance in the perseveration on LAI objects (Adjusted $\left.\mathrm{R}^{2}=0.190 ; \mathrm{F}(2,91)=11.87, \mathrm{p}<.01\right)$, with age $(\mathrm{p}<.001)$ but not group $(\mathrm{p}=.56)$ significantly contributing a unPIQue portion of the variance. The addition to the model of the age $\times$ group interaction contributed an additional $3 \%$ to the total variance $\left(\mathrm{R}^{2}\right.$ change $=$ $0.033)$, and the overall model remained statistically significant $(F(3,90)=9.46, p<.001)$. The incremental gain in variance was also significant $(\mathrm{F}(1,90)=3.89, \mathrm{p}=.05)$, indicating that the age $\times$ group interaction variable independently accounted for an additional $3 \%$ of the variance in perseveration on LAI objects above and beyond that of age and group alone.

\subsection{Detail-Orientation}

The combination of age and group significantly accounted for approximately $26 \%$ of the variance in detail-orientation across the entire task (Adjusted $\left.R^{2}=0.259\right)$, with age $(\mathrm{p}<$. $001)$ and group $(\mathrm{p}=.01)$ both offering statistically significant unique contributions to the variance. Adding the age $\times$ group interaction to the model explained an additional $.6 \%$ of the variance, which was not significant. 
For social images, age and group significantly predicted approximately $10 \%$ of the variance in detail-orientation (Adjusted $\left.\mathrm{R}^{2}=.096 ; \mathrm{F}(2,91)=5.95, \mathrm{p}<.01\right)$, with age $(\mathrm{p}<.01)$ but not group $(\mathrm{p}=.83)$ significantly contributing as a unique predictor. The addition of the age $\times$ group interaction explained another $2.5 \%$ of the variance, but was not significant. For object only arrays, age and group significantly accounted for approximately $22 \%$ of the variance in detail-orientation on HAI objects (Adjusted $=0.215 ; \mathrm{F}(2,91)=13.73$, $\mathrm{p}<.001$ ), with age $(\mathrm{p}<.001)$ and group $(\mathrm{p}=.05)$ contributing a unique portion of the variance. Inserting the age $\times$ group interaction did not predict any additional variance. Together, age and group did not predict a significant amount of the variance in detail-orientation on LAI object $(\mathrm{p}=.19)$, with neither age $(\mathrm{p}=.08)$ nor group $(\mathrm{p}=.48)$ acting as significant unique predictors. The addition of the age $\times$ group interaction explained an additional $2.8 \%$ of the variance, but was not significant.

\subsection{Supplemental Analyses}

PIQ did not significantly account for any of the variance in exploration (Adjusted $\mathrm{R}^{2}=$ $-0.014 ; \mathrm{F}(1,70)=.03, \mathrm{p}=.86)$. The addition of age and group accounted for an additional $33 \%$ of the variance $\left(\mathrm{R}^{2}\right.$ change $\left.=0.332\right)$, with age $(\mathrm{p}=.001)$ and group $(\mathrm{p}<.001)$ both significantly contributing as unique predictors above and beyond PIQ, and the overall model was now statistically significant (Adjusted $\mathrm{R}^{2}=0.303 ; \mathrm{F}(3,68)=11.31, \mathrm{p}<.001$ ). The addition of the age $\times$ group interaction explained an additional $2.2 \%$ of the variance, but this incremental gain was not significant $(\mathrm{p}=.14)$.

PIQ did not significantly account for any of the variance in perseveration (Adjusted $\mathrm{R}^{2}=$ $-0.008 ; \mathrm{F}(1,70)=1.56, \mathrm{p}=.22)$. The addition of age and group accounted for an additional $15 \%$ of the variance $\left(\mathrm{R}^{2}\right.$ change $\left.=0.154\right)$, with age $(\mathrm{p}<.01)$ and group $(\mathrm{p}<.05)$ both significantly contributing as unique predictors above and beyond PIQ, and the overall model was now statistically significant (Adjusted $\mathrm{R}^{2}=0.139 ; \mathrm{F}(3,68)=4.82, \mathrm{p}<.01$ ). The addition of the age $\times$ group interaction explained only an additional $.1 \%$ of the variance, and this incremental gain was not significant $(\mathrm{p}=.77)$.

PIQ did not significantly account for any of the variance in detail-orientation (Adjusted $\mathrm{R}^{2}=$ $-0.010 ; \mathrm{F}(1,70)=.30, \mathrm{p}=.58)$. The addition of age and group accounted for an additional $26 \%$ of the variance $\left(\mathrm{R}^{2}\right.$ change $\left.=0.261\right)$, with age $(\mathrm{p}=.001)$ and group $(\mathrm{p}=.001)$ both significantly contributing as unique predictors above and beyond PIQ, and the overall model was now statistically significant (Adjusted $\mathrm{R}^{2}=0.232 ; \mathrm{F}(3,68)=8.17, \mathrm{p}<.001$ ). The addition of the age $\times$ group interaction explained only an additional $1.8 \%$ of the variance, and this incremental gain was not significant $(\mathrm{p}=.21)$.

\section{Discussion}

Age was associated with significant increases in exploration and significant decreases in perseveration and detailed oriented scanning for both groups of children. In supplemental analyses with the $3 / 4 \mathrm{~s}$ of the sample that was cognitively characterized, these effects remained even after controlling for PIQ. Thus, the age-related patterns reported here appear to exist independent of level of intellectual functioning. Additionally, a significant interaction emerged suggesting that age differentially affects the number of images explored in children with autism and typically developing children. With age, typically-developing children explore more images within complex visual arrays. This aspect of visual attention was reduced in children with autism.

Our results suggest that the developmental difference for visual exploration is most pronounced with respect to the exploration of nonsocial information. The significant interaction between age and group for exploration on HAI objects demonstrates that children 
with autism exhibit less of an age-related increase in the exploration of these stimuli relative to TD comparison children due to a disproportionate attentional bias for these stimuli from very early in life, and thus may represent an early-emerging characteristic of the disorder. Considered in conjunction with the findings on total exploration patterns, an early attentional bias for nonsocial information (Klin et al., 2009; Pierce et al., 2011; Sasson et al., 2011) may be a primary feature of autism and its occurrence could potentially restrict the developmental acquisition of typical attention patterns.

These developmental differences in patterns of visual attention may have implications for the development and manifestation of the disparate features that comprise the syndrome of autism. Diminished exploration of social information could initiate a developmental sequence that could contribute to the emergent social deficits characteristic of autism (Klin et al., 2002). In addition, a bias for visually exploring nonsocial information could contribute to the development of restricted and perseverative patterns of behaviors and interests (Pellicano et al., 2011; Sasson et al., 2008). Thus, differences in the allocation of visual attention might help explain how two seemingly disparate characteristics of autism, social impairments and repetitive behavior, co-occur in the disorder.

Specific neurobiological mechanisms may underlie the disproportionate visual attention allocated toward specific categories of nonsocial information in autism. Investigations of reward networks suggest that the intrinsic reward value of social information signaled by the ventral striatum may be reduced in autism (Dichter et al., in press; Scott-Van Zeeland et al., 2010), and conversely, the reward value of specific nonsocial information categories may be increased (Dichter et al., in press). Furthermore, evidence suggests that in addition to evaluating the biological relevance of information in the environment, the amygdala may modulate attentional resources early in the information processing stream (Kennedy \& Adolphs, 2009; Phelps \& LeDoux, 2005). Atypical amygdala functioning could contribute to atypical prioritization of processing resources for social information observed in autism. Lastly, abnormal functioning in the dorsal striatum, which plays an important role in habitual learning as well as oculomotor control (Graybiel, 2008; Hikosaka et al., 2000), may serve a primary role in canalizing visual attention patterns. Future work that further refines our understanding of the neurobiological mechanisms underlying the disproportionate preference and prioritization of certain nonsocial aspects of the environment relative to social stimuli by individuals with autism is encouraged.

\subsection{Conclusions and Limitations}

We found that increased age was associated with disproportionately greater increases in visual exploration for typically developing children than for those children with autism. This finding suggests that the capacity to explore a complex array of visual information increases with age in typical development more so than in autism. The current study is limited in that we do not present associations with symptom severity, though the previous study by our group found associations (Sasson et al., 2008). While age and group effects remained when PIQ was considered in supplementary analyses with a subsample, this study is also limited by the incomplete characterization of cognitive level. Additionally, although cross-sectional studies can directly inform developmental models, longitudinal studies remain the gold standard for characterizing trajectories. Given the significant developmental differences in visual exploration which we observed, longitudinal studies of visual exploration in ASD appear warranted. These limitations notwithstanding, the current study demonstrates that abnormal visual exploratory behavior not only occurs very early in the development of ASD, but persists and may even increase with age. Thus, future research should examine how these differences in visual exploration relate to other aspects of the disorder and investigate whether it may be a viable target for examining outcome or predictors of early intervention in ASD. 


\section{Acknowledgments}

This research was funded by R01 MH073402 (Bodfish). JTE was supported by an NRSA award (5-T32HD007376) from NICHD to the Carolina Consortium on Human Development at the Center for Developmental Science, UNC. GSD was supported by K23 MH081285. Support was also provided by the Subject Registry Core of the Carolina Institute for Developmental Disabilities (P30 HD03110).

\section{References}

Dichter GS, Felder JN, Green SR, Rittenberg AM, Sasson NJ, Bodfish JW. Reward circuitry function in autism spectrum disorders. Social Cognitive and Affective Neuroscience. (in press).

Gauthier I, Skudlarski P, gore JC, Anderson AW. Expertise for cars and birds recruits brain areas involved in face recognition. Nature Neuroscience. 2000; 3:191-197.

Graybiel AM. Habits, rituals, and the evaluative brain. Annual Reviews of Neuroscience. 2008; 31:359-387.

Happe F, Frith U. The weak coherence account: detail-focused cognitive style in autism spectrum disorders. Journal of Autism and Developmental Disorders. 2006; 36:5-25. [PubMed: 16450045]

Hikosaka O, Takikawa Y, Kawagoe R. Role of the basal ganglia in the control of purposive saccadic eye movements. Physiological Reviews. 2000; 80:953-978. [PubMed: 10893428]

Johnson MH. Functional brain development in humans. Nature Reviews Neuroscience. 2001; 2:475483.

Kennedy DP, Adolphs R. Impaired fixation to eyes following amygdala damage arises from abnormal bottom-up attention. Neuropsychologia. 2010; 48:3392-3398. [PubMed: 20600184]

Klin A, Jones W, Schultz R, Volkmar F, Cohen D. Visual fixation patterns during viewing naturalistic social situations as predictors of social competence in individuals with autism. Archives of General Psychiatry. 2002; 59:809-816. [PubMed: 12215080]

Klin A, Lin DJ, Gorrindo P, Ramsay G, Jones W. Two-year-olds with autism orient to non-social contingencies rather than biological motion. Nature. 2009; 459:257-261. [PubMed: 19329996]

Landry R, Bryson SE. Impaired disengagement of attention in young children with autism. Journal of Child Psychology and Psychiatry. 2004; 45:1115-1122. [PubMed: 15257668]

Leppanen JM, Nelson CA. Tuning the developing brain to social signals of emotions. Nature Reviews Neuroscience. 2009; 10:37-47.

Lord C, Risi S, Lambrecht L, Cook EH, Leventhal BL, DiLavore PC, Pickles A, Rutter M. The autism diagnostic observation schedule-generic: a standard measure of social and communication deficits associated with the spectrum of autism. Journal of Autism and Developmental Disorders. 2000; 30:205-223. [PubMed: 11055457]

Lord C, Rutter M, Le Couteur A. Autism diagnostic interview-revised: a revised version of a diagnostic interview for caregivers of individuals with possible pervasive developmental disorders. Journal of Autism and Developmental Disorders. 1994; 24:659-685. [PubMed: 7814313]

Pellicano E, Smith AD, Cristino E, Hood BM, Briscoe J, Gilchrist ID. Children with autism are neither systematic nor optimal foragers. Proceedings of the National Academy of Sciences. 2011; 108:421-426.

Phelps EA, LeDoux. Contributions of the amygdala to emotion processing: from animal models to human behavior. Neuron. 2005; 48:175-187. [PubMed: 16242399]

Pierce K, Conant D, Hazin R, Stoner R, Desmond J. Preference for geometric patterns early in life as a risk factor for autism. Archives of General Psychiatry. 2011; 68:101-109. [PubMed: 20819977]

Pierce K, Courchesne E. Evidence for a cerebellar role in reduced exploration and stereotyped behavior in autism. Biological Psychiatry. 2001; 49:655-664. [PubMed: 11313033]

Risi S, Lord C, Gotham K, Corsello C, Chrysler C, Szatmari P, Cook EH, Leventhal BL, Pickles A. Combining information from multiple sources in the diagnosis of autism spectrum disorders. Journal of the American Academy of Child and Adolescent Psychiatry. 2006; 45:1094-1103. [PubMed: 16926617]

Sasson NJ. The development of face processing in autism. Journal of Autism and Developmental Disorders. 2006; 36:381-394. [PubMed: 16572261] 
Sasson NJ, Elison JT, Turner-Brown LM, Dichter GS, Bodfish JW. Circumscribed attention in young children with autism. Journal of Autism and Developmental Disorders. 2011; 41:242-247. [PubMed: 20499147]

Sasson NJ, Turner-Brown LM, Holtzclaw TN, Lam KSL, Bodfish JW. Children with autism demonstrate circumscribed attention during passive viewing of complex social and nonsocial picture arrays. Autism Research. 2008; 1:31-42. [PubMed: 19360648]

Scott-Van Zeeland AA, Dapretto M, Ghahremani DG, Poldrack RA, Bookheimer SY. Reward processing in autism. Autism Research. 2010; 3:53-67. [PubMed: 20437601]

Schultz RT. Developmental deficits in social perception in autism: the role of the amygdala and fusiform face area. International Journal of Developmental Neuroscience. 2005; 23:125-141. [PubMed: 15749240]

South M, Ozonoff S, McMahon WM. Repetitive behavior profiles in asperger syndrome and highfunctioning autism. Journal of Autism and Developmental Disorders. 2005; 35:145-158.

[PubMed: 15909401] 


\section{Highlights}

- Across development, typically developing children and individuals with autism spectrum disorders both show increased efficiency during visual exploration, but to different degrees.

- Individuals with autism spectrum disorders show a disproportionate attentional bias for certain categories of nonsocial information from an early age.

- Disproportionate visual attention to certain nonsocial objects may represent an early and persistent characteristic of autism spectrum disorders. 

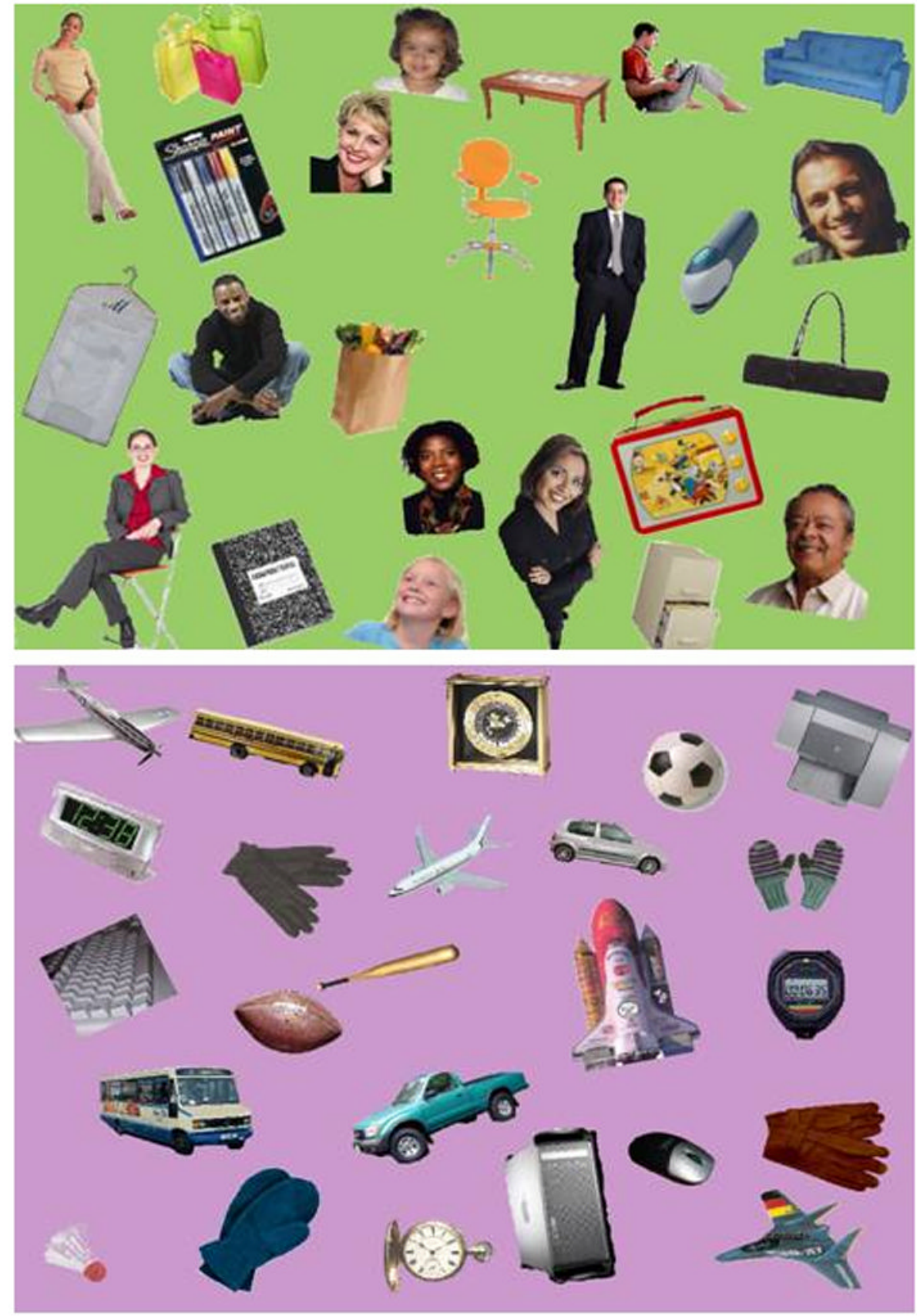

Fig. 1.

Examples of social + object and object arrays. 


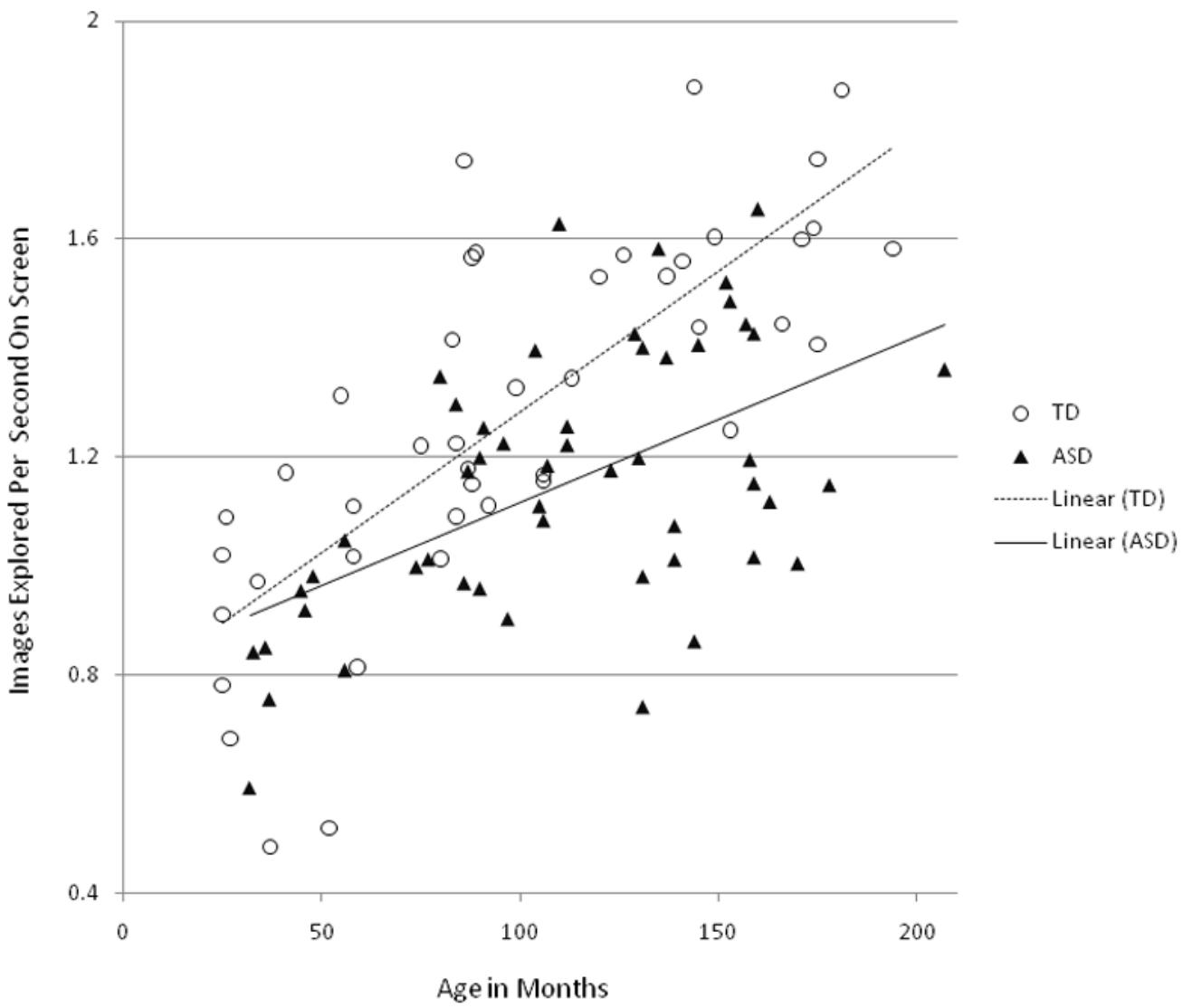

Fig. 2.

Age effects on visual exploration. 


\section{Table 1}

Demographic Features of Participants

\begin{tabular}{lll}
\hline Variable & $\begin{array}{l}\text { ASD group }(\mathbf{n}=51) \\
\text { Mean }(\text { SD })\end{array}$ & $\begin{array}{l}\text { TYP group (n=43) } \\
\text { Mean (SD) }\end{array}$ \\
\hline Age in months & $111.51(43.22)$ & $98.47(50.33)^{A}$ \\
Age range in months & $32-207$ & $25-194$ \\
Gender (\% male) & $90 \%$ & $91 \%$ \\
PIQ Estimate & $94.9(18.77)$ & $112.77(13.74)$ \\
Ethnicity (\% Caucasian) & $80 \%$ & $74 \%$ \\
\hline
\end{tabular}

PIQ available for 48 in ASD group and 30 in TYP group. All scores for children in TYP group and 42 in ASD are Leiter Brief IQ; 3 children in ASD group are Mullen Scales of Early Learning Nonverbal Developmental Quotient and 3 in ASD group are Bayley Scales of Infant

Development-2 ${ }^{\text {nd }}$ Edition Total Score.

Missing for 4 cases: 1 ASD and 3 TYP

$A_{\mathrm{p}>.05}$ 


\section{Table 2}

Correlation between age and visual attention variables for each group

\begin{tabular}{cc}
\hline & Age \\
\hline TD $(n=43)$ & \\
$\frac{\text { Exploration }}{\text { Overall }}$ & $0.771^{* *}$ \\
Social & $0.735^{* *}$ \\
HAI & $0.756^{* *}$ \\
LAI & $0.544^{* *}$
\end{tabular}

Perseveration

$\begin{array}{ll}\text { Overall } & -0.700^{* *} \\ \text { Social } & -0.467^{* *} \\ \text { HAI } & -0.692^{* *} \\ \text { LAI } & -0.546^{* *}\end{array}$

Detail-Orientation

$\begin{array}{cc}\text { Overall } & -0.626^{* *} \\ \text { Social } & -0.475^{* *} \\ \text { HAI } & -0.466^{* *} \\ \text { LAI } & -0.350^{*} \\ \text { ASD (n=51) }\end{array}$

Exploration

Overall $0.540^{* *}$

Social $0.473^{* *}$

HAI $\quad 0.364^{* *}$

LAI $\quad 0.457^{* *}$

Perseveration

Overall $-0.589^{* *}$

Social $-0.435^{* *}$

HAI $-0.599^{* *}$

LAI $-0.296^{*}$

Detail-Orientation

Overall $-0.385^{* *}$

Social $\quad-0.168$

HAI $-0.473^{* *}$

LAI $\quad-0.007$

Denotes $P<.05$

Denotes $P<.01$ 


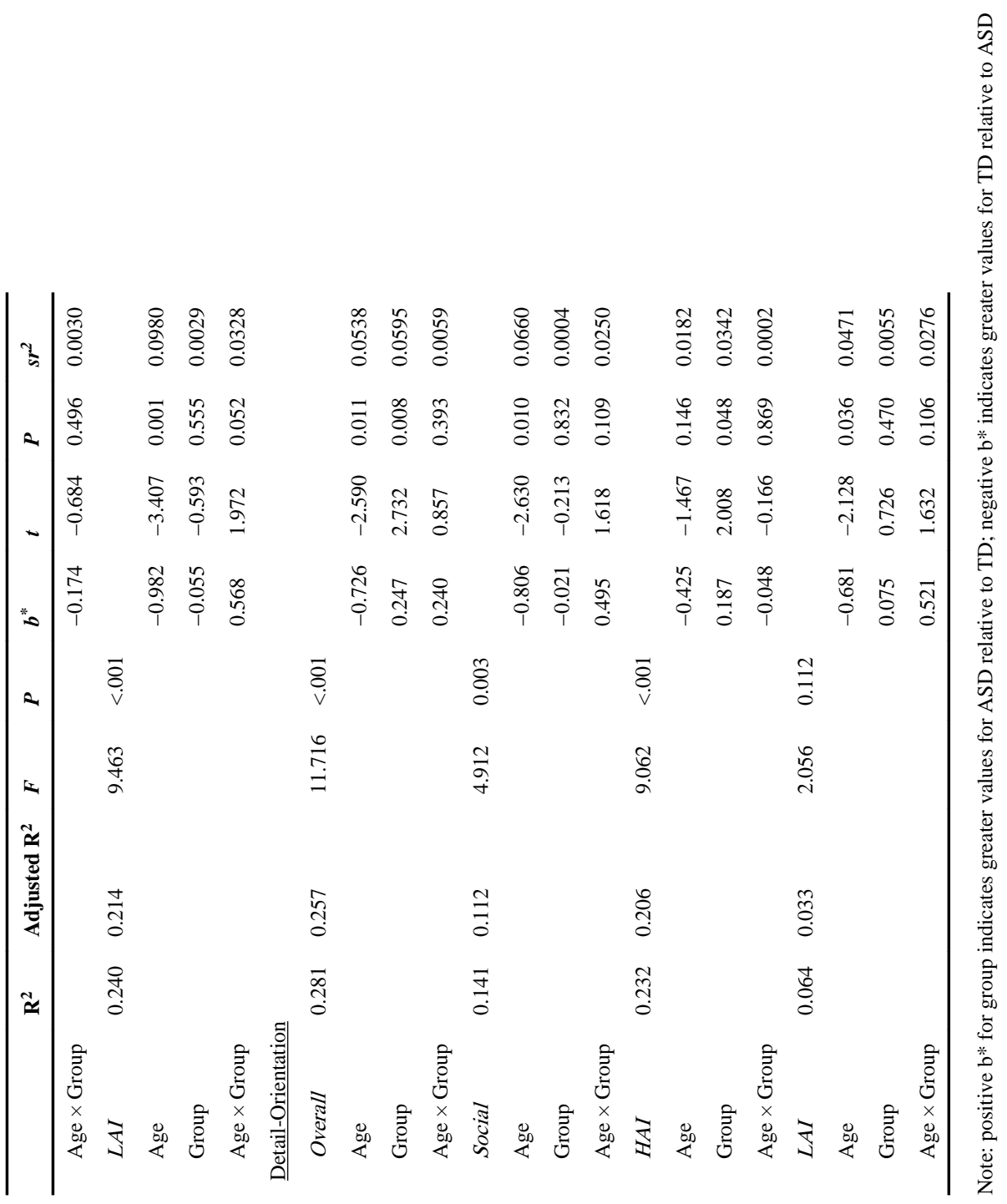

